\title{
Collaborative Evaluation of Early Design Decisions and Product Manufacturability
}

\author{
S. D. Kleban, W. A. Stubblefield, K. W. Mitchiner, J. L. Mitchiner, and M. Arms \\ Sandia National Laboratories \\ Albuquerque, New Mexico \\ \{sdkleba, wastubb, kwmitch, jlmitch, marms\}@,sandia.gov
}

\begin{abstract}
In manufacturing, the conceptual design and detailed design stages are typically regarded as sequential and distinct. Decisions made in conceptual design are often made with little information as to how they would affect detailed design or manufacturing process specification. Many possibilities and unknowns exist in conceptual design where ideas about product shape and functionality are changing rapidly. Few if any tools exist to aid in this difficult, amorphous stage in contrast to the many $C A D$ and analysis tools for detailed design where much more is known about the final product. The Materials Process Design Environment (MPDE) is a collaborative problem solving environment (CPSE) that was developed so geographically dispersed designers in both the conceptual and detailed stage can work together and understand the impacts of their design decisions on functionality, cost and manufacturability.
\end{abstract}

\section{Introduction}

The early design of complex artifacts often requires the collaboration of widely specialized, often geographically distributed, loosely coupled specialists. It is estimated that decisions made in the early design phase determine as much as 70 or 80 percent of the cost of a manufactured product [13]. Unfortunately, the skills, goals and activities involved in early design do not lead people to address the concerns of product manufacture. Often the knowledge needed to address such concerns is tentative, incomplete and spread across multiple points of view. This requires the use of heuristic knowledge to evaluate the impact of early design decisions on manufacturability. Most often, this heuristic knowledge resides in the skills of manufacturing process designers and engineers, people who are rarely involved in early design.

Current technologies enable us to construct collaborative environments to address this problem. These technologies include: advanced networking technology, the ability to store problem solving states and complex data as structured objects, highly expressive representations, knowledgebased advisors, the ability to deliver rich user interfaces to the desktop using Java and web browsers, cognitive theories of collaborative problem solving, and computer security.

We will present a CPSE, the Materials and Process Design Environment (MPDE), that addresses these problems in the context of mechanical design. MPDE uses a part-centric focus to coordinate the multiple points of view in the design team. It presents material scientists, mechanical engineers, process engineers and designers with an environment that integrates the representation of parts and assemblies with the tools, databases and advisors needed to evaluate and assess the impact of early design decisions on manufacturability and affordability. It allows users to save the state of a partial problem solution and share it with geographically distributed collaborators.

\section{Manufacturing Today}

There is and has always been a large gulf between designers and manufacturers during the conceptual design phase of a product. This gulf is the result of company culture, funding practices, and project focus. Typically in the conceptual phase, the emphasis is on geometry and potential performance. Materials selection is driven by performance needs, such as weight and durability, rather than by manufacturability and affordability. However, conceptual design determines as much as $70 \%$ of the cost of manufacturing the product (Fig. 1) [3]. Since manufacturing can amount to as much as $95 \%$ of the cost of the final product, lean manufacturing initiatives can only address $30 \%$ of those costs. The greatest opportunity to reduce product cost is in conceptual design, by addressing manufacturability and affordability in the product design itself. It is in this stage of the design that designers and engineers need tools to help them evaluate early design decisions for performance, manufacturability and cost.

In the past, manufacturability and manufacturing tools were normally ignored until the detailed design phase, where the designer had access to $2 \mathrm{D}$ or $3 \mathrm{D}$ representations of the product or part. The problem with doing all of this work in the detailed design phase, rather than in the conceptual phase, is that many decisions affecting manufacturability, particularly those involving 


\section{DISCLAIMER}

This report was prepared as an account of work sponsored by an agency of the United States Government. Neither the United States Government nor any agency thereof, nor any of their employees, make any warranty, express or implied, or assumes any legal liability or responsibility for the accuracy, completeness, or usefulness of any information, apparatus, product, or process disclosed, or represents that its use would not infringe privately owned rights. Reference herein to any specific commercial product, process, or service by trade name, trademark, manufacturer, or otherwise does not necessarily constitute or imply its endorsement, recommendation, or favoring by the United States Government or any agency thereof. The views and opinions of authors expressed herein do not necessarily state or reflect those of the United States Government or any agency thereof. 


\section{DISCLAIMER}

Portions of this document may be illegible in electronic image products. Images are produced from the best available original document. 


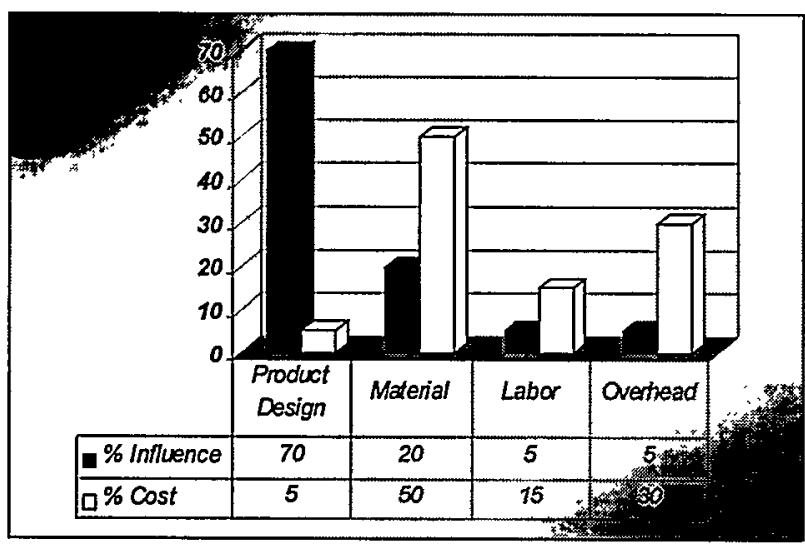

Figure 1. Cost Curve showing when in the product cycle manufacturing costs and influence are determined.

geometry, are frozen early in conceptual design. Rather than wait until this late stage, the designer needs to be able to make comparisons among processes to look at suitability, affordability, and risk, as soon as geometry features are being considered - often before detailed drawings or models of the part exist. Since manufacturing experts are typically not part of the conceptual design team, the team composition needs to change or the designer needs access to a "virtual manufacturing expert" to support his/her decisionmaking process.

The Smart Process Advisors in the MPDE provide product designers with a suite of "virtual manufacturing experts." MPDE has a two level architecture (figure 2). At the top are tools for conceptual design; from these, users can access detailed design tools. These tools help conceptual designers select materials, shaping processes and part joining processes, and evaluate the impact of these choices on manufacturability and cost. MPDE's conceptual design tools are:

i. The Materials selection advisor, which can be used to explore a broader variety of materials than may typically be known to the designer;

ii. The Near Net Shape Advisor [12], which enables the designer to determine his/her processing options (conventional vs. high speed machining, casting, laser freeform fabrication) as a function of geometry, manufacturability and cost;

iii. The Joining Advisor [9], which helps the designer understand what joining options (welding, brazing, soldering, solid state bonding, and mechanical fastening) are appropriate based on the material selected.

MPDE also provides detailed process design tools to help with the actual process specification. Currently, the only implemented detailed process design tool is the Welding Advisor [10], which advises the designer on the selection of a welding process and weld joint design.
While this tool appears to be more useful in the detailed design phase, the selection of a joint design can and does influence the geometry of the part and possibly the way the part needs to be processed. As a result, the designer may want to iterate back through the Near Net Shape Advisor. This tool can also be used in conceptual design, since it like the other Advisors does not require a 2D or 3D representation of the part. In addition, figure 2 shows proposed additional process design tools.

The Advisors ask the designer for geometric features, materials, and/or requirements that typically impact either manufacturability or cost. By specifying geometry at a high, often qualitative, level, it is simple to compare the effect of geometry on different processes: e.g. casting, machining, and laser freeform fabrication. For affordability considerations, parametric cost models are used. We have two types: (i) a simple parametric model based on number of parts, volume, material, and complexity and (ii) a commercial cost modeling code for DFM (Design For Manufacturing) [4].

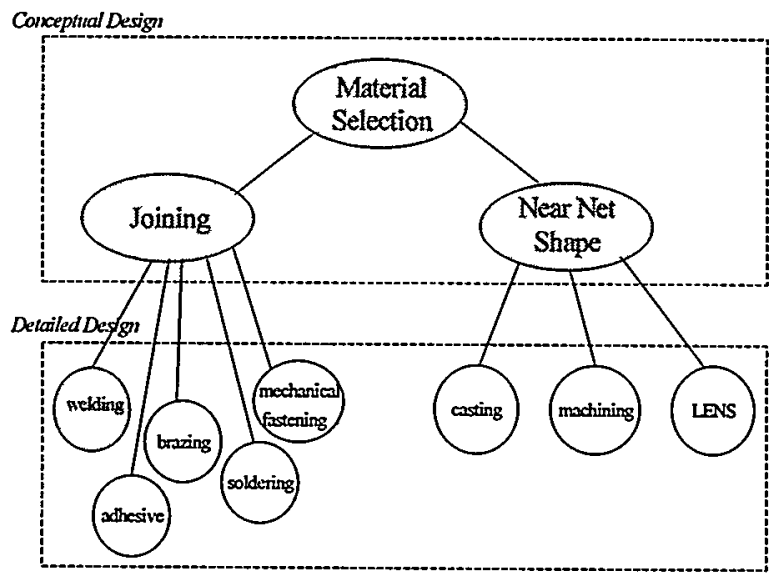

Figure 2. Two levels of architecture in the MPDE. The shaded tools are implemented in the MPDE (note: LENS = laser enabled net shaping = laser freeform fabrication)

\section{Collaboration and information sharing in early design}

In our view, early design begins with the initial discussions of a product's functionality and ends when its basic function, geometry and material have been at least tentatively specified. Although conceptual design has the greatest potential impact on the product's longterm success, it remains poorly understood and supported. Even though the last twenty years have seen enormous progress in the development of computer tools for the later stages of product design (CAD tools, configuration management systems, requirements tracking, etc), few tools exist to support conceptual REEREVED DEC 102090 
design. The few that currently exist or are in development address geometric and shaping considerations $[1,3,5,6]$ or functional analysis [2]. Indeed, the emergence of detailed solid models, project plans, formal information management and other project infrastructure is emblematic of the transition from early to late design.

Discussions of early design often treat it as a mystery of individual human creativity that is beyond systematic study. However, this "black art" follows a distinctive pattern that can be understood and used in the design of tools to support it. Characteristics of early design include:

1. Teams are only partially formed. As a rule, small teams of designers and engineers perform early product design. Manufacturing process engineers, reliability engineers, testing people and similar specialists typically join the team as the project moves into later stages. In many cases, there is not enough money in the early development ${ }^{\circ}$ budget for these specialists. If designers contact them at all, the manufacturing process engineer or other specialist acts as a consultant who is outside the core design team: their time and commitment to the project is limited.

2. Early design teams are closely coupled. Classical project management and planning is based on a well-structured series of activities specified in a work breakdown structure. In contrast, early. design seems to take place in small, tightly coupled teams, whose organization and interactions are not well structured. This is no accident: keeping teams small and co-located enhances the communication and flexibility required for creatively managing the problems of early design. For example, at Sandia National Laboratories, teams of less than a half-dozen people are common during Research and Development and other creative, highly conceptual design activities. These teams tend to be co-located, with team members often sharing offices, and rely on large amounts of unstructured communication. In later stages of product engineering and production, small teams give way to large, geographically distributed workgroups communicating in formal, highly structured ways. Unfortunately, this exacerbates the problems of bringing in additional expertise from such areas as manufacturing process design. In addition, few designers and engineers even realize that they need this expertise this early in design.

3. Early design decisions have greater impact on the overall project and product than decisions made later in the life cycle. Because little has been decided about the product's form and function in early design, each decision has tremendous leverage in reducing the range of possible product designs and manufacturing options. For example, designers usually choose a material early in design, based on performance, rather than manufacturability concerns. Selecting titanium as a material severely constrains later decisions about manufacturing, functionality and maintenance. Titanium's lightweight and great strength create opportunities for a product's geometry and functionality that stainless steel does not. It also constrains decisions about manufacture, reducing possibilities for joining or shaping processes.

4. Much information that is important in early design tends to resist formalization. Because so little is known about the product in early design, and because so many possibilities remain open to the design team, the processes and information used in early design resist formal characterization and offer little structure for coordinating group activity. Because later stages of design have gathered more information, projects have both greater need and ability to use formal design representations and the infrastructure that goes with them. Configuration Management systems, formal change control, requirements tracking and other forms of information infrastructure tend to be much more commonly used in late, rather than early, design (although they are probably just as important during conceptual phases). In early design, people tend to rely more on ad hoc representations (sketches, short memos, etc.) and communication (e-mail, hallway conversations, etc.). Although this does not eliminate either the possibility or usefulness of computer tools in early design, it underscores the importance of highly expressive, flexible representations and heuristic methods to these tools. It is important to develop flexible tools that capture the decision-making process during conceptual design.

5. There are few tools to support conceptual design. Product design typically begins with a general, often ill defined, set of requirements, and proceeds at a conceptual level until the rudiments of functionality, geometry, material and usability have been articulated. At this point, projects enter a detailed design phase, where all team members agree on the artifact's basic shape and function, and work to refine this design. Most work in CAD focuses on the detailed design phase, where methods, such as solid modeling, prototyping, testing, etc. become applicable.

6. Early design synthesizes multiple points of view. Before product design can move into the detailed phases, the design and engineering team must address a wide variety of issues in at least a high-level manner. Making early decisions on issues as diverse as geometry, function, material, manufacturing processes and product maintenance is more than a problem of gathering large amounts of information; it also requires a synthesis of different points of view. For example, both product and process engineers are concerned with material issues; however, process engineers must deal with the effect of 
material properties on things other than functionality: properties that may enhance functionality often complicate manufacture. For example, hardness may be desirable for product functionality, but choosing an excessively hard material may complicate machining. Early designers should (but often do not) evaluate all effects of such decisions. What makes it difficult is not just the complexity of the decisions: the knowledge involved in them is usually embodied in different people, with different agendas, schedules and points of view. Early design teams face more than a problem of organizing large amounts of information: they must also deal with different human agendas, assumptions and points of view.

7. Early design is opportunistic. Unlike later phases of design, decisions made in early design often effect radical changes in a product's geometry, material or function. Humans tend to respond to the underconstrained nature of early design by working at a high level of abstraction and focusing on what they perceive to be important issues. This gives early design an opportunistic flavor: designers seem to move unpredictably from one issue to another, one day emphasizing functional constraints, the next day focusing on material, etc. The opportunistic nature of early design accounts for the conventional distinction between "the art of design and the science of engineering." In contrast, detailed design has a more orderly flavor: most members of the team agree on what questions remain to be answered, on their relative importance and on the order in which they should be addressed. In this rapid, opportunistic milieu, decisions about manufacturability and maintenance are often ignored in favor of basic functionality, gross geometry and high-level material decisions.

\subsection{The structure of early design, and the requirements for a cooperative problem solving environment}

These observations underscore the need for Collaborative Problem Solving Environments to help manage the unique problems of early design and suggest a set of useful constraints on the functionality, interaction style, and information architecture of any early design tool. The importance of early design decisions and the need to synthesize diverse sets of information suggest a need for improved access to expert knowledge. The as yet poorly understood nature of early design activity and the narrow, specialized make-up of teams requires a rich, flexible collaborative problem solving environment, rather than a more rigid, workflow-oriented approach. The difficulty in formalizing early design information and processes suggests a reliance on rich, semantic representations and heuristic problem solving methods.

In particular, tools for conceptual design must:

1. Support access to geographically distributed information and collaborators, without disrupting the dynamics of small design teams. As discussed earlier, although the small, closely coupled nature of creative design teams is probably essential for conceptual design, this also promotes the risk of ignoring manufacturability, maintainability and other issues that are important to the product life cycle. On the other hand, efforts to encourage consideration of these factors must not disrupt the often-fragile dynamic of creative teams.

2. Help coordinate multiple points of view. Although different experts bring different points-ofview to the project, all of them must converge in a single product. Because there is often little consensus on the specific structure or function of products in early design, this agreement is often hard to achieve. Early design tools must present shared knowledge in a way that accommodates multiple points of view, goals and interpretations without necessarily tying the team to any one option.

3. Support non-deterministic activity. Tools to support early design must not restrict the opportunistic nature of this activity. Meaningful work decompositions are not available in early design; a work-break-down structure is one of the products of this design phase. Consequently, deterministic, sequential ways of coordinating work, such as work-flow engines, are not useful in early design.

4. Support both synchronous and asynchronous collaboration. The high-bandwidth communication required of creative teams involves both synchronous and asynchronous collaboration. This ranges from joint brainstorming sessions where all team members meet in a room to scribble on white-boards, to completely asynchronous communication using e-mail or memos. Typically, these modes of communication do not interact, nor do they support sharing of information between them.

5. Resist overly structuring product information. Although the demands of automation require imposing a structure on product information, we cannot ignore the flexibility required for early design. The answer to this is not to ignore structuring product information, but to do so at a high enough level of abstraction to allow the desired flexibility.

6. Exploit heuristic problem solving methods. In recent years, detailed design has come to exploit such mathematical, "science-based" approaches as finiteelement analysis. Early design decisions typically lack the formal basis these approaches require. Like human designers, conceptual design support systems must rely 
on - heuristic methods: experience-based, often qualitative, rules of thumb for making good decisions based on incomplete or unreliable information.

7. Tools must be easily extensible. Although extensibility is something of a mantra for contemporary software designers, it is more of an issue for early design tools than for their better understood counterparts. Our treatment of the structure of early design teams is, admittedly, fairly high level. We expect that our initial prototypes will reveal more information about this structure and demand changes to their successors.

In attempting to help designers pay closer attention to manufacturability and other late product life cycle issues in early design, MPDE addresses all of these requirements. They have influenced all aspects of its design, including the interaction style, the user interface, the information representation and the system architecture.

\subsection{The MPDE interaction style and user interface for cooperative problem solving}

In order to manage the complexity, ill-formedness and multiple points of view common to early design, the MPDE interface focuses on the structure of product information, rather than on the processes of product manufacture. The reason for this is simple: while the design team's understanding of manufacturing processes is usually incomplete and lacking in consensus, all members of the design team tend to agree on at least the product's basic function and gross geometry. In helping the design team to better evaluate manufacturing processes, MPDE organizes function and geometry according to their relationship to different components of the part. Rather than presenting users with a manufacturing workflow, it allows them to view specific processing phases (shaping, joining, etc.) organized by the part of the product they effect. This part focus serves several functions: it allows users to represent and reason about partially specified processes; it allows them to approach manufacturing process decisions in a flexible, opportunistic order; and it unifies the different points-ofview of product and process designers under the shared understanding of the artifact itself.

This part-focused interaction style supports both synchronous and asynchronous collaboration. A small group of designers may gather around the screen to use and evaluate the system jointly. Alternatively, a group or individual may store results for later use by a colleague. In addition, this style supports the documentation of decisions and the reasoning behind the choices being made. Finally, the part/joint focus supports integration of different points of view. Although product designers and process engineers may view requirements and manufacturing processes differently, the basic structure of the product forms a common point of reference from which to make decisions. Product information is given a simple top-level structure: a product is an assembly of parts and joints.

Figure 3 shows the MPDE's top-level screen and illustrates this taxonomy. The left-hand panel allows entry to the MPDE and also provides access to additional tools. Although not integrated into the MPDE system, these tools can be used in either the conceptual design phase or later in the detailed design phase.

The main panel (the lower, right hand area of the screen) is the heart of the MPDE. It presents the user with a set of parts and joints. A part consists of a material, one or more shaping processes, and additional processing steps, such as joining or welding. A joint consists of two parts: a joining process and additional information about the joining process. For example, a welded joint may include information about the weld geometry and weld schedule.

Next to each list are icons representing tools for designing or analyzing various properties of parts or joints. Currently, MPDE includes a materials selection system for selecting a part's material given high-level functional and process requirements and a Near-Net Shape Process Selection Advisor for choosing a basic shaping process. For joints, MPDE provides a Materials and Joining Advisor to help select a joining process for a given pair of materials and a set of high-level function constraints on the joint. If the joint is a weld, the SmartWeld Advisor helps determine the welding process, joint geometry and other particulars of the weld.

Each tool helps the user with one or more design decisions. For example, the Materials Selection System helps them choose a part material. After running the advisor, the material is recorded in the part record. Similarly, after running the Near Net Shape Advisor, the desired shaping process is recorded with the part. It also records the relative cost of the process in comparison with the other processes being assessed. This approach allows the user maximum flexibility in the order of decision-making, and supports the opportunistic searching that is typical of early design. For example, although knowing the material is important to choosing a shaping process, the user may still use the Near Net Shape Advisor and enter a value of unknown for material. Although this compromises the quality of the result because it does not take into account producibility from a materials perspective, the user may still desire to do so in early design. Later, after choosing a material, the designer may revisit the Near Net Shape Advisor.

MPDE represents parts and joints at a very high level of abstraction: a part is a shape and a material; a joint 


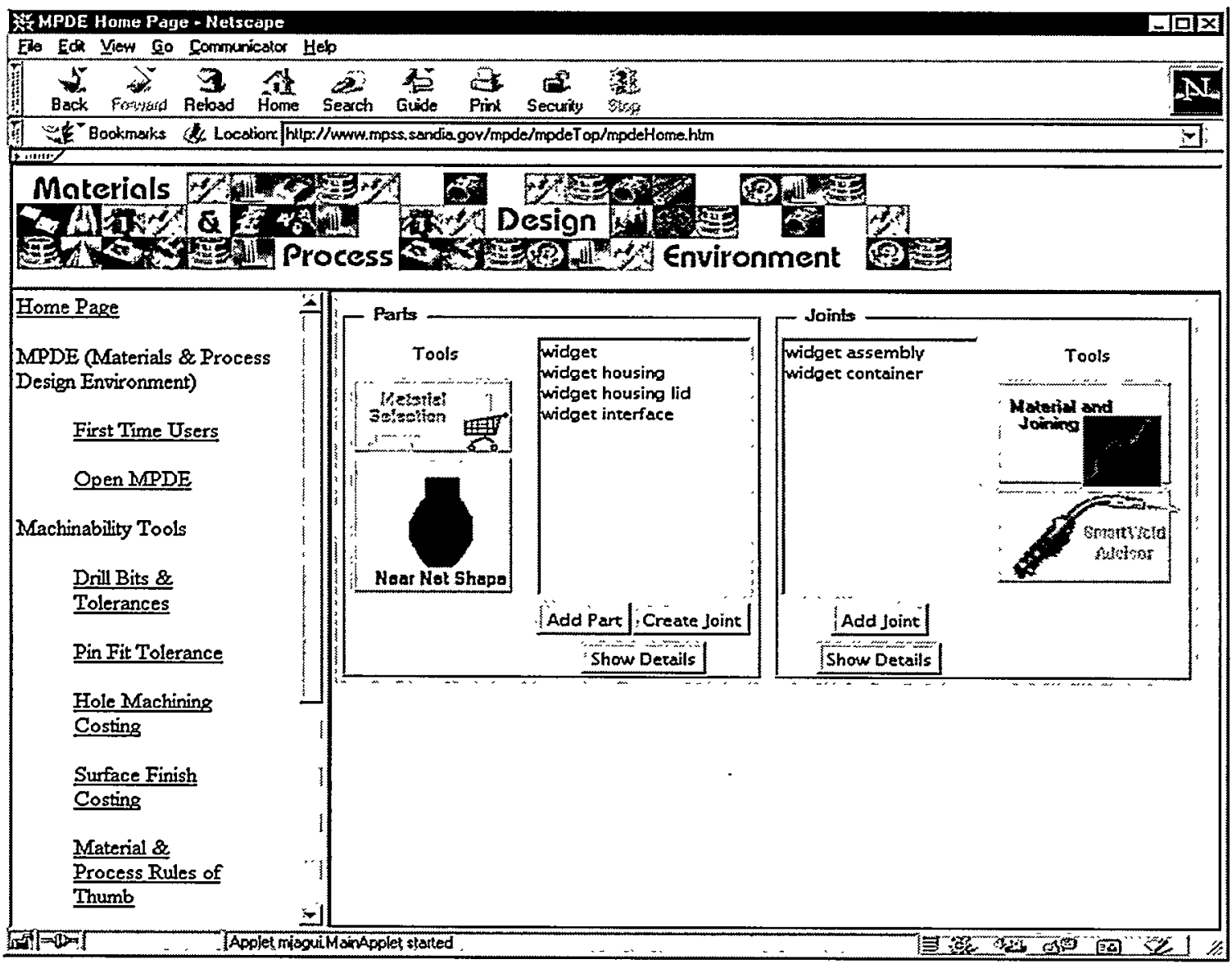

Figure 3. Screen image of MPDE Homepage.

involves two parts and a joining process. In addition, part geometry is treated at a high level of abstraction rather than requiring an explicit 2-D or 3-D drawing of the geometry. For example, the Near Net Shape Advisor (see below for details) allows a user to state that a part has a curved surface, a certain number of pockets and protrusions and general complexity metric. It will make a heuristic determination of a shaping process based on this information. By not requiring a solid model or other detailed geometric representation, the tool supports brainstorming and "what if" evaluations.

\subsection{MPDE's tools and advisors}

This commitment to the needs of conceptual design activities is continued in MPDE's tools and advisors. To illustrate, consider a typical interaction. A designer begins by defining a part. This definition does not require a drawing or any other information about the part: the user may simply name and save the part. Assume the next decision is to choose a material.
Selecting the part and clicking on the Material Selection icon opens the Materials Selection System.

The Materials Selection System categorizes materials properties into four groups: Mechanical, Electrical, Thermal and General. Selecting a property displays the distribution of property values across all $(2000+)$ materials known to the system. The histogram displays the number of materials in different value ranges for each property. In addition, the histogram allows users to move lower and upper bounds on the desired range of values and instantly see the number of materials in that range.

This selection mechanism addresses the needs of conceptual designers in two ways: 1) it allows them to specify a range of property values, rather than specific values, and 2) it allows them to manage sets of candidate materials. The Materials Selection System maintains a working set of materials. This set is the intersection of all the sets of materials that fall in the specified range of each selected property value. Once the user has specified a set of candidate materials, he/she may either 
choose one material or save the entire set of candidate materials for future evaluation.

In our scenario, assume the user chooses a set of materials that meet broad cost and performance constraints and would next like to evaluate the impact of different materials on shaping process selection. $\mathrm{He} / \mathrm{she}$ may save the entire set of candidate materials with the part, and then open the Near Net Shape Process Selection Advisor (NNS) (Figure 4). On detecting that the chosen part has a set of candidate materials, NNS will create a list of $<$ Process, Material $>$ pairs for every combination of processes and materials. Currently, NNS "knows about" machining, three types of investment casting processes and Laser freeform fabrication (LENS). LENS is a direct fabrication process that uses a laser to selectively melt powdered metal into a shape specified by a 3-D CAD model.

NNS then queries the user for additional information about part geometry, desired lot size and lead time, general tolerance data and process efficiency issues. A set of tests uses this information to evaluate each
$<$ material, process $>$ pair. Figure 4 shows one of the data entry screens for the advisor. The left hand side of the screen lets the user select different categories of part data. The center screen allows the user to enter part constraints. The screen in the figure shows one of three sets of geometry queries. Note that it does not require a drawing of the part but lists features that have an impact on manufacturability and cost and asks the user how many of these features exist in the part. Although this simple representation does not account for the location of features on the part, or interactions between them, it does allow a "first-cut" at evaluating the impact of geometry on each <material, process $>$ pair.

At any time during the evaluation process, the user may view a summary of all evaluations for all <material, process $>$ pairs. Figure 5 shows this evaluation screen. The table ranks each <material, process> pair by total score, which is the weighted sum of its scores on all suitability tests. In addition, the histogram at the bottom of the screen shows the comparative scores for the highest scoring material for each process. Green (light

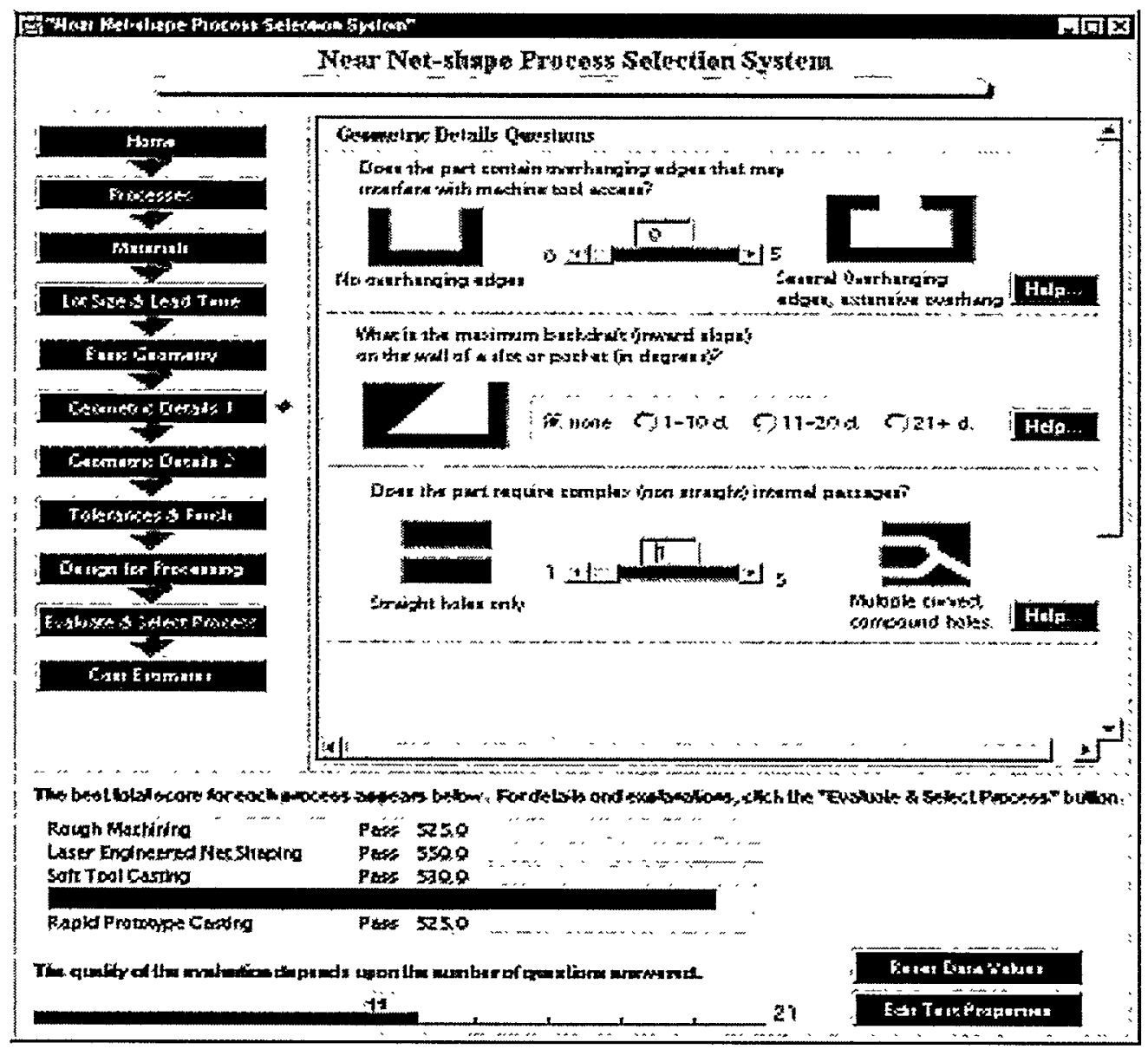

Figure 4. Geometric Detail Questions in the Near Net Shape Process Selection Advisor. 
grey in figure) indicates that the <process, material> passes all of the requirements. Yellow (white) indicates that the <process, material> fail one or more desired (soft) requirements, and red (dark grey) indicates that the <process, material> fail one or more hard requirements.

Like the Materials Selection System, the Near Net Shape Advisor addresses the particular needs of early design. It allows the user to evaluate the effect of geometry on manufacturing process by listing critical features, rather than requiring a solid model. It also maintains and evaluates a large set of process and material combinations. Finally, after ranking the possible process and material combinations, it allows the user to either select one or a set of possibilities for further evaluation.

The remaining tools, the Materials and Joining Advisor and the SmartWeld welding advisor use similar techniques to support the needs of designers in the early stages of conceptual design.

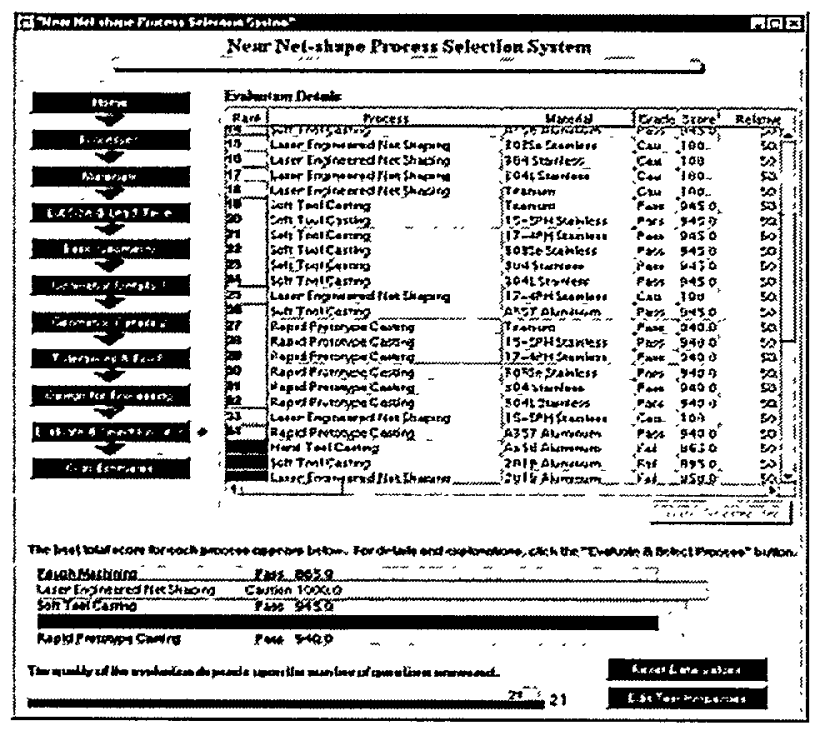

Figure 5. Evaluation Results ranked in tabular form from most recommended to least recommended

\section{Architecture}

The architecture of our CPSE reflects the way collaborators in manufacturing design perform their work. Their geographic separation and ad hoc methods of communication require a system architecture that allows and provides for asynchronous interaction with manufacturing designs. Product designers need a virtual space where they can come to collaborate on product designs when needs arise. Based on our fieldwork, product designers tend to work from their desktop computer. They want a CPSE delivered to their desktop, instead of going down the hall to a meeting room or entering some virtual world that takes them out of the flow of their daily work. These requirements, together with the collection of conceptual design software tools that run on multiple computers, led to a distributed computing architecture.

Desktop access to distributed computing resources has been an active area of research for decades but has recently become mainstream with the advent of the Internet. Database access, financial calculators, and maps with driving directions are common Internet tools delivered to the desktop through a web browser. Providing this type of access to manufacturing design tools is a goal of the architecture. Numerous technologies are available for distributed computing, but selecting the correct collection and combining them into a coherent architecture is a challenging problem. The manufacturing design tools that need to be integrated into a common environment were developed over years. As a result, they were implemented in different languages and run on different platforms. Since commercial tools also need to be integrated, the system must provide the ability to add, change, and remove tools over time as necessary. The architecture must be flexible and easily extensible since new design tools are constantly being developed.

\subsection{SmartWeld}

In the early to mid 1990s, Sandia developed SmartWeld [11], a collaborative welding design and analysis system. Many of its goals were similar to the system described here, but its focus was exclusively on welding. The user interface presented a guided environment where each step in the design and analysis process had to be completed sequentially. It began with a welding advisor that recommended a weld joint design and weld process based on the functional requirements for the joint. It then invoked a CAD tool to visualize the new joint design. Next, it generated a mesh and used a thermal finite element analysis code to determine the heat flow through the materials. Finally, a visualization system allowed the user to view a movie of the heat flow during the weld. Although narrow in its scope, SmartWeld addressed many of the technical and interface issues involved in supporting collaborative design. These included the development of representations to integrate diverse tool sets and the presentation of diverse processes in a coherent interface.

SmartWeld also made us aware of the need for a distributed computing architecture but the technologies in the early nineties were somewhat limiting. CORBA was still being developed and the Internet was in its childhood. Consequently, we used a tightly coupled framework to integrate the collection of welding tools. 
Tightly coupled in this sense means a set of tools that communicated through hard coded, low level mechanisms such as sockets. Although this is appropriate for well defined, stable systems, this tightly coupled framework is inflexible and difficult to extend.

\subsection{Loosely Coupled Frameworks}

Loosely coupled frameworks allow easy insertion, modification, and deletion of software components. They consist of distributed objects that communicate through object message passing instead of processes communicating through byte streams, as with sockets. This technology is fairly recent, starting with CORBA in the early 1990s and followed by COM/DCOM and Java's RMI. More recent mechanisms such as Java's EJB and CORBA3 are just becoming available. At the lowest level, all distributed computing uses processes which communicate over sockets, but loosely coupled frameworks provide a more natural object-oriented abstraction, a framework infrastructure such as registration/naming services and security, and multithreaded servers where numerous clients can connect to a single server. These are desirable characteristics for a dynamic and uncertain environment where the tools are constantly being added and upgraded.

\subsection{MPDE Architecture}

The loosely coupled MPDE architecture evolved from the diversity of existing distributed materials and process selection components. We developed these components during the same years where computer languages (namely Java) and platforms evolved; consequently, early components were written in $\mathrm{C}$ or $\mathrm{C}++$ with the later ones written in Java. There was a need to integrate $\mathrm{C} / \mathrm{C}++$ with Java, Java with Java, and Java with databases. In addition, file management systems and mechanisms were required to store and modify product and process information. With so many interacting components, and the need to support system evolution, we recognized a need for a variety of distributed technologies. Figure 6 shows the client and the types of network connections and protocols used to access each server.

Several design tools (currently, the Materials Selection and the Materials and Joining Advisor) access the materials database using JDBC (Java DataBase Connection). For both of these advisors, we are currently using the Cambridge Materials Database [7] marketed by Granta Design in England. While numerous materials databases exist, this one has proved particularly useful because of the completeness of data with respect to both properties and manufacturability. When performing searches, no material will be omitted because of missing data on a property that is included in the search criteria.

The entire user interface for the MPDE is written as a Java applet and accessed over the network through a web browser. Using Java applets gave us the platform independence we wanted. This was desirable because our users had both PCs with Windows NT and Suns with Solaris. The graphical user interface (GUI) is

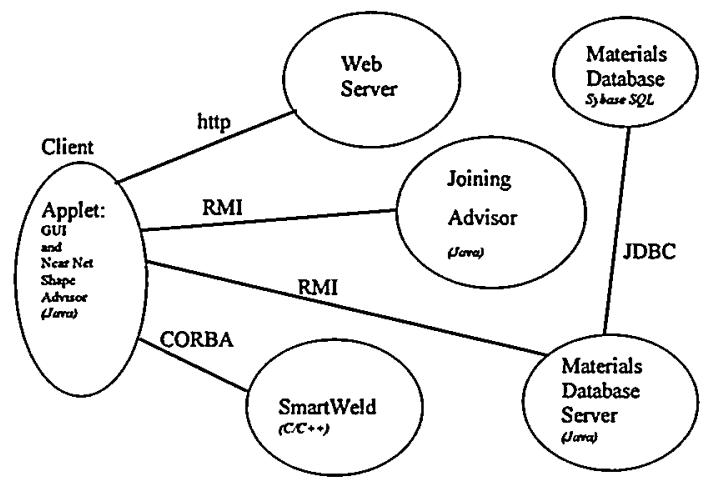

Figure 6. The distributed components in the MPDE and their communication protocols.

lightweight in that all the functionality of the MPDE is provided from the server or network of servers. The GUI simply provides the menus, text boxes/input fields, graphics, etc., with the databases and knowledge-based tools residing on the servers. The exception is the Near Net Shape Process Selection Advisor, which is currently part of the applet. There was not really a compelling reason to make this a server. The knowledge-based rules run very quickly and the need for database access did not exist since the material selection has already been completed and stored locally as a working dataset. Standard Java security restricts Java applets from accessing the local disk and making connections to servers other than to the web server from which the applet is delivered. To overcome this, we had to use the signing mechanism [8] provided for Java applets.

RMI enables communication from the applet to the servers where the database and advisors reside. RMI is a convenient technology when the distributed components are all written in Java. The Materials Database Server and the Joining Advisor were both written in Java and thus accessed by RMI.

CORBA is used for communication between the applet and the SmartWeld Advisor. Since SmartWeld is written in $\mathrm{C} / \mathrm{C}++$, and the applet in Java, CORBA was the logical choice because of the ease in which components written in different languages can communicate. They use a common language, CORBA's Interface Definition 
Language (IDL), to define their interfaces and any CORBA enabled programming language can communicate through the common IDL.

\subsection{MPDE Ontology}

In order for a number of components or tools to interact and cooperatively problem solve, they need an ontology, i.e. a common understanding and representation of the objects and relations in the world of discourse. An ontology is a formal specification of a conceptual system. In MPDE, the specification is accomplished through concepts and their relations and the conceptualization is the design and manufacturing domain. In manufacturing and design, the ontology can quickly become very complex, describing both products and manufacturing processes to great detail. Since the MPDE is used in conceptual design many of the details are not necessary.

In designing the MPDE ontology, we wanted a simple, intuitive formulation that could be easily extended. The basic entities are parts, materials, and joints. The shaping processes include laser freeform fabrication, casting, and machining; the joining processes include soldering, welding, mechanical fastening, brazing, solid state bonding and adhesive bonding. Currently, our representation of parts captures their material, their processing constraints (lot size, lead-time) and a highlevel, qualitative geometry description. Joints define a relationship between two parts and specify a joining process and details of the process implementation (e. g. a weld schedule). We believe that by keeping the number of basic entities small, focusing on the physical structure of assemblies, and remaining fairly abstract, the MPDE ontology will allow integration of additional information as the system grows, without needing frequent reformulation of the taxonomy.

\section{Conclusion}

Perhaps MPDE's most important contribution has been as a tool for exploring the domain of conceptual design support. Because the environment for conceptual design is so ill-defined, we found that designers were not prepared to provide a clear set of requirements for the tools we were developing. The best approach was to work in an iterative fashion, presenting user interfaces, providing prototypes, and reworking versions. This iterative approach also addresses the cultural change issues inherent in introducing collaborative environments into a creative, closely-knit human activity. Working with engineers on a series of prototypes, not only brings hidden cultural problems to the forefront, but also gives them an opportunity to help solve these issues and take ownership of the results.

\section{References}

[1] Abel, C. A., Edwards, K. L., and Ashby, M. F. (1994). "Materials, processing and the environment in engineering design: the issues." Materials \& Design 15(4): 179-193.

[2] Ashby, M. F. (1993). "Materials Selection in Mechanical Design." La metallurgia italiana vol. 86, n. 10.

[3] Boothroyd, G., Dewhurst, P. and Knight, W. A. (1991). "Selction of Materials and Processes for Component Parts." International Journal of Advanced Manufacturing Technologies. 6:98-111, London: Springer-Verlag.

[4] Galorath Incorporated (1999). SEER-DFM Software.

[5] Giachetti, R. (1997). "A Decision Support Systems for Material and Manufacturing Process Selection," Journal of Intelligent Manufacturing, January.

[6] Dargie, P. P., Parmeshwar, K., and Wilson, W. R. D. (1982). "MAPS-1: computer-aided design system for preliminary material and manufacturing selection." ASME Transactions 104:126-136.

[7] Granta (1994). Granta Design Limited, 20 Trumpington Street, Cambridge CB2 1QA, United Kingdom.

[8] Java/Certificates (1998). Java Security, Scott Oaks, O'Reilly \& Associates, USA.

[9] Kleban, S. D. (1998). "Concurrent Materials and Process Selection in Conceptual Design." Proceedings Artificial Intelligence and Manufacturing Research Planning Workshop, 98-102. Albuquerque, New Mexico: AAAI Press.

[10] Kleban, S. D. (1996). "Design Issues of a Knowledgebased Welding Advisor." Proceedings Artificial Intelligence and Manufacturing Research Planning Workshop, 93-96. Albuquerque, New Mexico: AAAI Press.

[11] Mitchiner, J. L., Kleban, S. D., Hess, B. V., Mahin, K. W., and Messink, D. (1996). "SmartWeld: A Knowledge-based Approach to Welding." Proceedings Artificial Intelligence and Manufacturing Research Planning Workshop, 129-135. Albuquerque, New Mexico: AAAI Press.

[12] Rivera, J. J., Stubblefield, W. A., Ames, A. L. (1996). "Critices and Advisors: Heuristic Knowledge and Manufacturability." Proceedings Artificial Intelligence and Manufacturing Research Planning Workshop, 146-152. Albuquerque, New Mexico: AAAI Press.

[13] Ullman, D. G. (1992). The Mechanical Design Process, McGraw Hill, New York.
Sandia is a multiprogram laboratory operated by Sandia Corporation, a Lockheed Martin Company, for the United States Department of Energy under contract DE-AC04-94AL85000. 